\title{
Bosonic Memory Channels
}

\author{
Vittorio Giovannetti ${ }^{1}$ and Stefano Mancini ${ }^{2}$ \\ ${ }^{1}$ NEST-INFM 83 Scuola Normale Superiore, I-56126 Pisa, Italy. \\ ${ }^{2}$ Dipartimento di Fisica, Università di Camerino, I-62032 Camerino, Italy.
}

(Dated: November 11, 2018)

\begin{abstract}
We discuss a Bosonic channel model with memory effects. It relies on a multi-mode squeezed (entangled) environment's state. The case of lossy Bosonic channels is analyzed in detail. We show that in the absence of input energy constraints the memory channels are equivalent to their memoryless counterparts. In the case of input energy constraint we provide lower and upper bounds for the memory channel capacity.
\end{abstract}

PACS numbers: 03.67.Hk, 03.65.Ud, 89.70.+c

Quantum communication with continuous alphabet provides an interesting alternative to the traditional discrete alphabet based approach (using e.g. qubits) [1]. Much effort has been recently devoted to characterize continuous alphabet quantum channels in terms of information capacities 2]. At present capacity results are only known for a restricted class of channels. Among them the lossy Bosonic channel, which consists of a collection of Bosonic modes that lose energy en route from the transmitter to the receiver. The classical capacity of such communication lines has been found to be unaffected by the use of entanglend inputs among different channel uses 3], in close analogy to what happens for a wide class of qubit alphabet channels [4]. However, for the latter it was argued that entangled inputs may enhance the information transmission in the presence of correlated noise (memory) 5]. Increasing attention has been dedicated to memory effects in quantum channels, but only within discrete alphabets [6, 7].

Here, we investigate the memory effects in continuous alphabet channels. In Sec. Iwe present a class of Bosonic memory channels which in the case of $n$ unconstrainedinputs, is unitarily equivalent to its i.d.d. memoryless 2 counterpart with an input space $n$ times larger than the single use case. Unfortunately such equivalence is partially lost when imposing energy constraint [8, 9, 10] on the input states of the communication line. Under such conditions in Sec. III we supply some bounds for the capacity of these channels. The papers ends with the conclusions in Sec. III]

\section{MODEL}

A quantum channel that uses continuous alphabet can be modeled by a Bosonic field mode whose phase space quadratures enable for continuous variable encoding/decoding [8]. On $n$ uses of such a channel we have to consider $n$ independent Bosonic modes, described by annihilation operators $a_{k}$ for $k=1, \cdots, n$. As depicted in Fig. 11 we restrict the analysis to the case where each $a_{k}$ interacts with an environment mode $b_{k}$ through a beam splitter of transmittivity $\eta \in[0,1]$, thus modeling lossy channels. The signal-noise coupling is then characterized

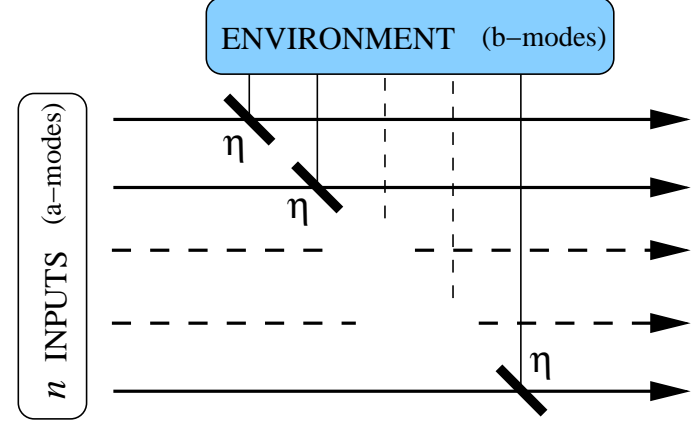

FIG. 1: (Color online) Scheme of the communication scenario: $n$ uses of the lossy Bosonic channel correspond to $n$ input Bosonic modes $a_{k}$ interacting with the environment modes $b_{k}$ trough $n$ beam splitters.

by $U \equiv \otimes_{k=1}^{n} U_{k}$ with

$$
U_{k}=\exp \left[\left(a_{k}^{\dagger} b_{k}-a_{k} b_{k}^{\dagger}\right) \arctan \sqrt{\frac{1-\eta}{\eta}}\right],
$$

the unitary operator which satisfies the following transformations 11

$$
\begin{aligned}
U_{k} a_{k} U_{k}^{\dagger} & =\sqrt{\eta} a_{k}-\sqrt{1-\eta} b_{k}, \\
U_{k} b_{k} U_{k}^{\dagger} & =\sqrt{\eta} b_{k}+\sqrt{1-\eta} a_{k} .
\end{aligned}
$$

Let $r$ be the density matrix in the Hilbert space $\mathcal{H}_{\text {tot }}^{(n)} \equiv$ $\otimes_{k=1}^{n} \mathcal{H}_{k}$ which describes the input state of the $n$ channel uses. Here $\mathcal{H}_{k}$ is the Hilbert space associated with input mode $a_{k}$. For a memoryless channel the environment acts independently on each $a_{k}$. This can be described by assuming the modes $b_{k}$ to be in the same state $\rho_{b}$. The output density matrix corresponding to $r$ is hence given by $[12]$

$$
\mathcal{L}(r)=\operatorname{Tr}_{b}\left[U\left(r \otimes r_{b}\right) U^{\dagger}\right],
$$

where the trace is performed over the environment's degrees of freedom, initially in the state $r_{b} \equiv \rho_{b}^{\otimes n}$. Because of the tensorial structure of $U$ and $r_{b}$, the map (3) becomes

$$
\mathcal{L}(r)=\otimes_{k=1}^{n} \ell_{k}(r)
$$


with $\ell_{k}$ being the map on $\mathcal{H}_{k}$ associated with the $k$-th channel use which transforms the density matrix $\rho$ of $\mathcal{H}_{k}$ according to

$$
\ell_{k}(\rho) \equiv \operatorname{Tr}_{b_{k}}\left[U_{k}\left(\rho \otimes \rho_{b}\right) U_{k}^{\dagger}\right] .
$$

A memory channel is characterized by non trivial correlations between the environment actions on the different channel uses which cannot be accounted for by Eq. (4). We model this situation by replacing the separable state $r_{b}$ of Eq. (3) with the entangled state

$$
\tilde{r}_{b} \equiv \Omega_{b} r_{b} \Omega_{b}^{\dagger},
$$

where $\Omega_{b}$ is a unitary, multi-mode squeezing operator [1]

$$
\Omega_{b} \equiv \exp \left[\sum_{k, k^{\prime}}\left(\xi_{k k^{\prime}}^{*} b_{k} b_{k^{\prime}}-\xi_{k k^{\prime}} b_{k}^{\dagger} b_{k^{\prime}}^{\dagger}\right)\right]
$$

which couples the $b_{k}$ modes through the squeezing parameters $\xi_{k k^{\prime}}$. The corresponding output state of the channel is hence described by the map

$$
\tilde{\mathcal{L}}(r)=\operatorname{Tr}_{b}\left[U\left(r \otimes \tilde{r}_{b}\right) U^{\dagger}\right] .
$$

The dependence of Eq. (8) on $n$ is generally more involved with respect to that of Eq. (4). Equation (8) also depends on the parameters $\xi_{k k^{\prime}}$ and for for $\xi_{k k^{\prime}}=0$ it is $\Omega_{b}=$ $\mathbb{1}$ and $\tilde{\mathcal{L}}=\mathcal{L}$. It is worth noting that in defining the memory channel model (8) it is not necessary to assume Eqs. (1) and (7). As a matter of fact, $U_{k}$ can be any unitary operator that couples the $k$-th channel use mode with its noise $b_{k}$, while $\Omega_{b}$ can be any unitary operator which introduces correlations between the $b_{k}$. We will focus on the case described by Eqs. (11) and (7) since here an interesting simplification occurs.

Our aim is now to relate the memory channel of Eq. (8) to the memoryless channel of Eq. (3). Let us consider

$$
\Omega \equiv \exp \left[\sum_{k, k^{\prime}}\left(\xi_{k k^{\prime}}^{*} a_{k} a_{k^{\prime}}-\xi_{k k^{\prime}} a_{k}^{\dagger} a_{k^{\prime}}^{\dagger}\right)\right]
$$

which represents a multi-mode-squeezing (unitary) operator acting on the inputs mode $a_{k}$ with the same squeezing parameters $\xi_{k k^{\prime}}$ of (7). Defining the density matrix

$$
\tilde{r} \equiv \Omega^{\dagger} r \Omega,
$$

and using Eq. (6) we rewrite Eq. [8] as

$$
\tilde{\mathcal{L}}(r)=\operatorname{Tr}_{b}\left[U\left(\Omega \otimes \Omega_{b}\right)\left(\tilde{r} \otimes r_{b}\right)\left(\Omega^{\dagger} \otimes \Omega_{b}^{\dagger}\right) U^{\dagger}\right] .
$$

The transformations (2) can be used to verify that

$$
U\left(a_{k} a_{k^{\prime}}+b_{k} b_{k^{\prime}}\right) U^{\dagger}=a_{k} a_{k^{\prime}}+b_{k} b_{k^{\prime}},
$$

which shows that $\Omega \otimes \Omega_{b}$ commutes with $U$. Therefore Eq. (11) yields

$$
\tilde{\mathcal{L}}(r)=\Omega \operatorname{Tr}_{b}\left[U\left(\tilde{r} \otimes r_{b}\right) U^{\dagger}\right] \Omega^{\dagger},
$$

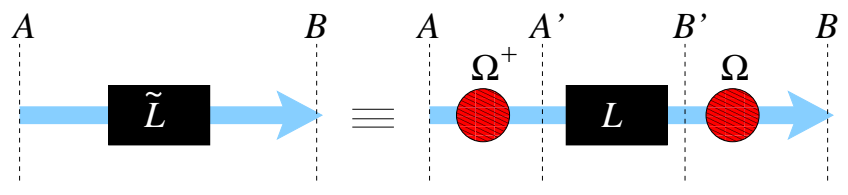

FIG. 2: (Color online) Decomposition of $\tilde{\mathcal{L}}$ of Eq. (8). Input states enter the system in $A$ (input) and leave it in $B$ (output). According to Eq. (14) we can identify two intermediate steps: in $A^{\prime}$ the input state has been transformed by the unitary operator $\Omega^{\dagger}$ and enters the map $\mathcal{L}$; in $B^{\prime}$ it is finally transformed by $\Omega$.

where: i) since $\Omega$ does not act on $b_{k}$, we have moved it out of the trace operation, ii) since $\Omega_{b}$ is unitary we have used the cyclicity of the trace to eliminate it. Notice that, apart from the unitary operator $\Omega$, the right-hand side of Eq. (13) is a standard memoryless Bosonic channel (3) which couples the input state $\tilde{r}$ with the environment state $r_{b}$; thus we can write Eq. (13) as

$$
\tilde{\mathcal{L}}(r)=\Omega \mathcal{L}(\tilde{r}) \Omega^{\dagger}
$$

This equation shows that the map $\tilde{\mathcal{L}}$ can be decomposed in the following three operations (see also Fig. 2):

1) apply the anti-squeezing operator $\Omega^{\dagger}$ to the input state $r$;

2) send the resulting state in the channel $\mathcal{L}$;

3) squeeze the final state with $\Omega$.

Notice that, if the noise parameters $\xi_{k k^{\prime}}$ are known to the communicating parties, the unitary operators $\Omega^{\dagger}$ and $\Omega$ at 1) and 3) can always be included in the encoding and decoding stages of the transmission. In this sense, $\tilde{\mathcal{L}}$ and $\mathcal{L}$ are unitarily equivalent and one expects their ability in transferring information (classical or quantum) to be the same.

\section{CONSTRAINED INPUTS}

The "equivalence" of $\tilde{\mathcal{L}}$ and $\mathcal{L}$ is partially broken in the case of constrained inputs [8]. However also in this case, Eq. (14) can be used to relate the capacities of these two channels. Let us consider for example the capacity of the memoryless channel when $\rho_{b}$ 's of Eq. (3) are thermal states with average photon number $M$, i.e.

$$
\rho_{b} \equiv \frac{1}{M+1}\left(\frac{M}{M+1}\right)^{b_{k}^{\dagger} b_{k}} .
$$

Under the hypothesis that the inputs states $r$ of the channel $\mathcal{L}$ have less then $N$ photons per channel use,

$$
\operatorname{Tr}\left[r \sum_{k=1}^{n} a_{k}^{\dagger} a_{k}\right] \leqslant n N,
$$


it is believed [3, 9, 10] that the classical capacity $C(\mathcal{L}, N)$ of $\mathcal{L}$ can be saturated by using Gaussian encodings. These allow one to achieve a transmission rate equal to

$$
G(\mathcal{L}, N)=n[g(\eta N+(1-\eta) M)-g((1-\eta) M)]
$$

where

$$
g(x)=(x+1) \ln (x+1)-x \ln x,
$$

and where the linear dependence on $n$ is a consequence of the absence of memory effects in the transmission. Even though the identity $C(\mathcal{L}, N)=G(\mathcal{L}, N)$ has been proved [3] only for $M=0$ (environment's vacuum state), there are strong evidences that it should also apply for $M>0$.

\section{A. Upper bounds}

In the following we derive two independent upper bounds for the maximum number of classical information $C(\tilde{\mathcal{L}}, N)$ that can be reliably transmitted through the $n$ uses of the memory channel $\tilde{\mathcal{L}}$ when its inputs $r$ are constrained by Eq. (16).

Equation (14) establishes that transmitting $r$ into $\tilde{\mathcal{L}}$ is equivalent to transmitting $\tilde{r}$ of Eq. (10) into $\mathcal{L}$. The maximum average photon number $\bar{N}$ per channel use associated with the latter state can be computed using the transformations (2). In particular, for $r$ satisfying Eq. (16) one can show that

$$
\operatorname{Tr}\left[\tilde{r} \sum_{k=1}^{n} a_{k}^{\dagger} a_{k}\right] \leqslant n \bar{N}
$$

where

$$
\bar{N}=N[\cosh (4 \bar{d})+\sinh (4|\bar{d}|)]+s_{1}+s_{2} \geqslant N .
$$

In the above expression $s_{1}$ and $s_{2}$ are positive quantities defined in Appendix A 1 and $\bar{d}$ is the eigenvalue of the of the $n \times n$ matrix $\xi_{k k^{\prime}}$ (assumed real symmetric for the sake of simplicity) having maximum absolute value. The quantity $\bar{N}$ determines the maximum value of average photon number per channel use that is entering the channel $\mathcal{L}$ at point $A^{\prime}$ of Fig. 2 when we feed the channel $\tilde{\mathcal{L}}$ with $N$ photons per use. We can exploit this fact to conclude that the capacity $C(\tilde{\mathcal{L}}, N)$ cannot be greater than the capacity $C(\mathcal{L}, \bar{N})$ of the memoryless channel $\mathcal{L}$ with $\bar{N}$ average photon number per channel use, i.e.

$$
C(\tilde{\mathcal{L}}, N) \leqslant C(\mathcal{L}, \bar{N}) .
$$

Clearly this inequality does not depend on the validity of the conjecture [3, 9, 10]. However, in order to derive an explicit expression for the bound (21) it is useful to assume [3, 9, 10] and evaluating the right-hand side term of (21) by means of the function $G(\tilde{\mathcal{L}}, N)$ of Eq. (17), i.e.

$$
C(\tilde{\mathcal{L}}, N) \leqslant n[g(\eta \bar{N}+(1-\eta) M)-g((1-\eta) M)] .
$$

An alternative upper bound for $C(\tilde{\mathcal{L}}, N)$ can be obtained by fixing $n$ and by assuming the corresponding map $\tilde{\mathcal{L}}$ to represent a memoryless channel. This allows us to derive the following inequality 13

$$
C(\tilde{\mathcal{L}}, N) \leqslant \sup _{m} C_{m}\left(\tilde{\mathcal{L}}^{\otimes m}, N\right) / m
$$

where $m$ is the number of successive uses of the "memoryless" channel $\tilde{\mathcal{L}}$ and where 14 ]

$$
\begin{aligned}
C_{m}\left(\tilde{\mathcal{L}}^{\otimes m}, N\right) \equiv \max _{p^{(i)}, R^{(i)} ; N}\left\{S\left(\tilde{\mathcal{L}}^{\otimes m}(R)\right)\right. \\
\left.-\sum_{j} p^{(i)} S\left(\tilde{\mathcal{L}}^{\otimes m}\left(R^{(i)}\right)\right)\right\}
\end{aligned}
$$

is the maximum amount of information the two communicating parties can share by feeding with probabilities $p^{(i)}$ the $m$ copies of $\tilde{\mathcal{L}}$ with messages $R^{(i)} \in\left(\mathcal{H}_{\text {tot }}^{(n)}\right)^{\otimes m}$. Here $S(R)=-\operatorname{Tr}[R \ln R]$ is the von Neumann entropy and $R=\sum_{i} p^{(i)} R^{(i)}$ is the average input of $\tilde{\mathcal{L}}^{\otimes m}$. The maximization in Eq. (24) is performed over all ensembles $\left\{p^{(i)}, R^{(i)}\right\}$ which, for each $\tilde{\mathcal{L}}$, satisfy the energy constraint (16), i.e.

$$
\operatorname{Tr}\left[R\left(\sum_{k=1}^{n} a_{k}^{\dagger} a_{k}\right)^{\otimes m}\right] \leqslant m n N .
$$

Likewise Refs. [3, 10] we provide an upper bound for (24) by replacing the first/second term at the righthand side with the maximum/minimum respectively

$$
C_{m}(\tilde{\mathcal{L}}, N) \leqslant m \max _{r ; N}\{S(\tilde{\mathcal{L}}(r))\}-\min _{R}\left\{S\left(\tilde{\mathcal{L}}^{\otimes m}(R)\right)\right\} .
$$

The subaddittivity of the von Neumann entropy has been used to transform the maximization over $R \in\left(\mathcal{H}_{\text {tot }}^{(n)}\right)^{\otimes m}$ into maximization over inputs $r$ of $\mathcal{H}_{\text {tot }}^{(n)}$, and the constraint (25) has been dropped in the minimization. Equation (26) establishes that $C_{m}(\tilde{\mathcal{L}}, N)$ can be bounded by the difference between the maximum output entropy of single use $(m=1)$ of $\tilde{\mathcal{L}}$ and the minimum output entropy of the $m$ channel uses: let us compute these quantities.

For inputs $r$ that satisfy the constraint (16), Eqs. (2) and (8) establish that the maximum average photon number we can get at the output of the channel $\tilde{\mathcal{L}}$ is equal to $n N_{\text {out }}$ where,

$$
N_{\text {out }}=\eta N+(1-\eta)\left(s_{0} M+s_{1}\right)
$$

with $s_{1}$ as in Eq. (20) and

$$
s_{0} \equiv \sum_{j=1}^{n} \cosh \left(4 d_{j}\right) / n \geqslant 1,
$$

(see Appendix A3 for details). We can hence upper bound the output entropy of $\tilde{\mathcal{L}}$ with $n$ times the entropy 
$g\left(N_{\text {out }}\right)$ of a thermal state whose total average photon number is equal to $N_{\text {out }}[\underline{3}]$.

To compute the minimum output entropy of the channel $\tilde{\mathcal{L}}$ we use a conjecture proposed in Ref. [12]. In fact, from Eq. (14) and the invariance of $S$ under unitary operations, we have

$$
\min _{R}\left\{S\left(\tilde{\mathcal{L}}^{\otimes m}(R)\right)\right\}=\min _{R}\left\{S\left(\mathcal{L}^{\otimes m}(R)\right)\right\}
$$

According to the analysis of Ref. 12] the minimum output entropy of the channel $\mathcal{L}$ should be provided by vacuum input: this result has not been proven yet but, as in the case of the conjecture Eq. (17), there is strong evidence in support of it (as a matter of fact these two conjectures are strongly related). Assuming the conjecture of Ref. 12] we can simplify Eq. (29) as follows,

$$
\min _{R}\left\{S\left(\tilde{\mathcal{L}}^{\otimes m}(R)\right)\right\}=m n g((1-\eta) M) .
$$

which replaced in Eq. (26) and (23) gives,

$$
\begin{gathered}
C(\tilde{\mathcal{L}}, N) \leqslant n\left[g\left(\eta N+(1-\eta)\left(s_{0} M+s_{1}\right)\right)\right. \\
-g((1-\eta) M)] .
\end{gathered}
$$

The right-hand sides of Eqs. (22) and (31) are two independent upper bounds for the capacity of the $n$ successive uses of the memory channel $\tilde{\mathcal{L}}$. They have been derived by assuming the conjectures discussed in Refs. [3, 9, 10] and Ref. [12], respectively. Both of them are greater or equal to the alleged capacity $G(\mathcal{L}, N)$ of Eq. (17) of a memoryless channel $\mathcal{L}$ with average constraint $N$ (it follows for instance from the fact that $g(x)$ is an increasing function of $x$ ).

\section{B. Lower bound}

A lower bound for $C(\tilde{\mathcal{L}}, N)$ can be obtained by providing an encoding-decoding procedure that allows to achieve reliable information transfer. This is not a simple task for a memory channel. However we can use the decomposition rule (14) to transform encodings of $\mathcal{L}$ (which are simpler to characterize) into encodings of $\tilde{\mathcal{L}}$.

The only known encoding that allows the memoryless channel $\mathcal{L}$ to asymptotically achieve the transmission rate (17) requires the sender to feed the channel with thermal states [3, 9, 10]. Suppose that she/he manages to produce a thermal state at point $A^{\prime}$ of Fig. 2 and assume that the average photon number of such state is $N^{\prime}$. This means that the state of the $n$ modes in $A^{\prime}$ is given by

$$
\tilde{r}=\bigotimes_{k=1}^{n} \frac{1}{N^{\prime}+1}\left(\frac{N^{\prime}}{N^{\prime}+1}\right)^{a_{k}^{\dagger} a_{k}} .
$$

The corresponding state in $A$ is obtained by inverting the relation Eq. (10) and has average photon number equal to

$$
\operatorname{Tr}\left[\Omega \tilde{r} \Omega^{\dagger} \sum_{k} a_{k}^{\dagger} a_{k}\right]=n\left(s_{0} N^{\prime}+s_{1}\right),
$$

with $s_{0}$ and $s_{1}$ as in Eqs. (20) and (27) (see Appendix A2 for details). Since we are allowed to supply less then $N$ average photon number per channel use, we should require

$$
s_{0} N^{\prime}+s_{1} \leqslant N, \quad \Longrightarrow \quad N^{\prime} \leqslant \frac{N-s_{1}}{s_{0}} \leqslant N .
$$

For all $N^{\prime}$ satisfying the above relation the sender is able to use the optimal encoding (32) to transfer messages with capacity $G\left(\mathcal{L}, N^{\prime}\right)$ given in Eq. (17). This means that for large enough $n$, the following inequality holds

$$
C(\tilde{\mathcal{L}}, N) \geqslant G\left(\mathcal{L},\left(N-s_{1}\right) / s_{0}\right) .
$$

Since $C(\mathcal{L}, N)$ is always greater than the right-hand side of Eq. [35], we cannot claim that $C(\tilde{\mathcal{L}}, N)$ is definitely greater than $C(\mathcal{L}, N)$.

\section{CONCLUSIONS}

We have discussed a model of quantum memory channel employing continuous alphabets which relies on the use of multi-mode squeezed (entangled) environment state. In the simple case of lossy Bosonic channel we have found a unitarily equivalence (14) between the map $\tilde{\mathcal{L}}$ of the memory channel and the map $\mathcal{L}$ of its memoryless counterpart. When no constraints on the input states there is a perfect equivalence in the ability of the these channels in transferring information. As a consequence, entangled inputs can only be used to reach an optimal encoding, but they do not improve the channel's performance. This shows that the role of entanglement is subtle. In particular, it seems no longer useful when other unlimited resources are available. In the more realistic scenario of energy constrained input states, we provided upper and lower bounds for the capacity of the memory channel. In particular, from Eqs. (21) and (35) we have

$$
G\left(\mathcal{L},\left(N-s_{1}\right) / s_{0}\right) \leqslant C(\tilde{\mathcal{L}}, N) \leqslant C(\mathcal{L}, \bar{N}),
$$

which, assuming the conjecture [3, 9, 10], shows that the classical capacity of the memory channel is bounded by classical capacities of the memoryless channel $\mathcal{L}$ having different power constraints. It is worth noticing that, because of Eq. (14), the above relation generalizes also to all the other capacities (e.g. quantum capacity, entanglement assisted capacity [2]) of $\tilde{\mathcal{L}}$ and $\mathcal{L}$.

Finally, we believe that the results presented here, though not giving a conclusive answer on the usefulness of entanglement versus memory effects, are deep enough. Furthermore, the presented model is fairly general and could be used to study a variety of specific and practical 
situations. For instance it could be interesting to analyze the case where Eq.(7) only connects nearest neighbors modes, that is, each use is only affected by the previous one. As such this work paves the way for further studies in Bosonic memory channels.

\section{APPENDIX A}

Define $Z$ the $n \times n$ matrix whose elements are $\xi_{k k^{\prime}}$ of Eq. (9). Without loss of generality we can choose $Z$ to be symmetric. For the sake of simplicity, in the following we will also assume $Z$ to be Hermitian. If $Z$ is symmetric and Hermitian it is also real and we can diagonalize it by means of a unitary matrix $V$ of real element $v_{k k^{\prime}}$, i.e.

$$
Z=V \cdot D \cdot V^{T} \Longrightarrow \xi_{k k^{\prime}}=\sum_{j=1}^{n} d_{j} v_{k j} v_{k^{\prime} j}
$$

where $D$ is a $n \times n$ diagonal matrix with real elements $d_{j}$. Using the above relation one can verify that

$$
\sum_{k k^{\prime}} \xi_{k k^{\prime}} a_{k} a_{k^{\prime}}=\sum_{j} d_{j} c_{j} c_{j}
$$

where for all $j=1, \cdots, n$

$$
\begin{aligned}
c_{j} & =\sum_{k} v_{k j} a_{k}, \\
a_{k} & =\sum_{j} v_{k j} c_{j} .
\end{aligned}
$$

Notice that the operator $c_{j}$ form a set of independent annihilation operators which satisfy the usual commutation relations,

$$
\left[c_{j}, c_{j^{\prime}}\right]=0 \quad\left[c_{j}, c_{j^{\prime}}^{\dagger}\right]=\delta_{j j^{\prime}},
$$

and the identity

$$
\sum_{j} c_{j}^{\dagger} c_{j}=\sum_{k} a_{k}^{\dagger} a_{k}
$$

Replacing the relation (A4) in (9) we get,

$$
\begin{aligned}
\Omega & \equiv \exp \left[\sum_{j} d_{j}\left(c_{j}^{2}-\left(c_{j}^{\dagger}\right)^{2}\right)\right] \\
& =\bigotimes_{j=1}^{n} \exp \left[d_{j} c_{j}^{2}-d_{j}\left(c_{j}^{\dagger}\right)^{2}\right] .
\end{aligned}
$$

Thus the operator $\Omega$ squeezes the mode $c_{j}$ as follows,

$$
\begin{aligned}
& \Omega c_{j} \Omega^{\dagger}=\cosh \left(2 d_{j}\right) c_{j}+\sinh \left(2 d_{j}\right) c_{j}^{\dagger}, \\
& \Omega^{\dagger} c_{j} \Omega=\cosh \left(2 d_{j}\right) c_{j}-\sinh \left(2 d_{j}\right) c_{j}^{\dagger} .
\end{aligned}
$$

\section{Derivation of Eq. (19)}

From Eq. (14) we know that transmitting the state $r$ into $\tilde{\mathcal{L}}$ is equivalent to transmitting the state $\tilde{r}$ of Eq. (10) into $\mathcal{L}$. Equations (A6) and (A8) allow us to compute the average photon number of $\tilde{r}$ as follows

$$
\begin{gathered}
\operatorname{Tr}\left[\tilde{r} \sum_{k=1}^{n} a_{k}^{\dagger} a_{k}\right]=\operatorname{Tr}\left[r \Omega\left(\sum_{k=1}^{n} a_{k}^{\dagger} a_{k}\right) \Omega^{\dagger}\right] \\
=\operatorname{Tr}\left[r \Omega\left(\sum_{j=1}^{n} c_{j}^{\dagger} c_{j}\right) \Omega^{\dagger}\right] \\
=\operatorname{Tr}\left[r \left(\sum_{j=1}^{n} \cosh \left(4 d_{j}\right) c_{j}^{\dagger} c_{j}\right.\right. \\
\left.\left.+\sinh \left(4 d_{j}\right)\left(c_{j}^{\dagger} c_{j}^{\dagger}+c_{j} c_{j}\right) / 2\right)\right]+n s_{1},
\end{gathered}
$$

where

$$
s_{1} \equiv \sum_{j=1}^{n} \sinh ^{2}\left(2 d_{j}\right) / n \geqslant 0 .
$$

Equation (A9) can be upper bounded using the following inequalities,

$$
\begin{aligned}
& \sum_{j=1}^{n} \cosh \left(4 d_{j}\right) \operatorname{Tr}\left[r c_{j}^{\dagger} c_{j}\right] \leqslant \cosh (4 \bar{d}) \operatorname{Tr}\left[r \sum_{j=1}^{n} c_{j}^{\dagger} c_{j}\right] \\
& =\cosh (4 \bar{d}) \operatorname{Tr}\left[r \sum_{k=1}^{n} a_{k}^{\dagger} a_{k}\right] \leqslant n N \cosh (4 \bar{d}), \quad(\mathrm{A} 11)
\end{aligned}
$$

where $\bar{d}$ is the eigenvalues $d_{j}$ with maximum absolute value. In deriving the above expression we used (A6) and the fact that $r$ has at most $N$ average photon number per channel uses. Analogously we have

$$
\begin{aligned}
& \frac{1}{2} \sum_{j=1}^{n} \sinh \left(4 d_{j}\right) \operatorname{Tr}\left[r\left(c_{j}^{\dagger} c_{j}^{\dagger}+c_{j} c_{j}\right)\right] \\
& \leqslant \frac{1}{2} \sum_{j=1}^{n} \sinh \left(4\left|d_{j}\right|\right) \operatorname{Tr}\left[r\left(2 c_{j}^{\dagger} c_{j}+1\right)\right] \\
& \leqslant n N \sinh (4|\bar{d}|)+n s_{2},
\end{aligned}
$$

with

$$
s_{2}=\sum_{j=1}^{n} \sinh \left(4\left|d_{j}\right|\right) /(2 n) \geqslant 0 .
$$

Equation (19) finally follows by replacing (A11) and (A12) in A9.

\section{Derivation of Eq. (33)}

Here we compute the average photon number associated with the state $\Omega \tilde{r} \Omega^{\dagger}$ with $\tilde{r}$ the thermal state 
defined in (32). We proceed as in Eq. (149) obtaining

$$
\begin{aligned}
\operatorname{Tr}[ & \left.\Omega \tilde{r} \Omega^{\dagger} \sum_{k} a_{k}^{\dagger} a_{k}\right]=\operatorname{Tr}\left[\tilde { r } \left(\sum_{j=1}^{n} \cosh \left(4 d_{j}\right) c_{j}^{\dagger} c_{j}\right.\right. \\
& \left.\left.-\sinh \left(4 d_{j}\right)\left(c_{j}^{\dagger} c_{j}^{\dagger}+c_{j} c_{j}\right) / 2\right)\right]+n s_{1} \\
& =\sum_{j=1}^{n} \cosh \left(4 d_{j}\right) N^{\prime}+n s_{1} .
\end{aligned}
$$

where the last identity follows from (A3) and from the properties

$$
\begin{aligned}
\operatorname{Tr}[\tilde{r} & \left.a_{k}^{\dagger} a_{k^{\prime}}\right]=\delta_{k k^{\prime}} N^{\prime} \\
\operatorname{Tr}\left[\tilde{r} a_{k} a_{k^{\prime}}\right] & =0 .
\end{aligned}
$$

\section{Derivation of Eq. (27)}

The average photon number at the output of the channel $\tilde{\mathcal{L}}$ associated with the input $r$ can be computed using the relation

$$
\begin{aligned}
U^{\dagger} a_{k}^{\dagger} a_{k} U= & \eta a_{k}^{\dagger} a_{k}+(1-\eta) b_{k}^{\dagger} b_{k} \\
& +\sqrt{\eta(1-\eta)}\left(b_{k}^{\dagger} a_{k}+a_{k}^{\dagger} b_{k}\right)
\end{aligned}
$$

and the result of the previous section. In particular from the definition (8) we have

$$
\begin{array}{r}
\operatorname{Tr}_{a}\left[\tilde{\mathcal{L}}(r) \sum_{k=1}^{n} a_{k}^{\dagger} a_{k}\right]=\eta \operatorname{Tr}_{a, b}\left[r \otimes \tilde{r}_{b} \sum_{k=1}^{n} a_{k}^{\dagger} a_{k}\right] \\
+(1-\eta) \operatorname{Tr}_{a, b}\left[r \otimes \tilde{r}_{b} \sum_{k=1}^{n} b_{k}^{\dagger} b_{k}\right] \\
\quad+\sqrt{\eta(1-\eta)} \operatorname{Tr}_{a, b}\left[r \otimes \tilde{r}_{b} \sum_{k=1}^{n}\left(b_{k}^{\dagger} a_{k}+a_{k}^{\dagger} b_{k}\right)\right] .
\end{array}
$$

The first term on the right-hand side is proportional to the input average photon number of $r$. The second and the third instead can be computed as in Eq. A14.

\section{ACKNOWLEDGMENTS}

VG gratefully acknowledges useful discussions with N. J. Cerf and for informing him of his recent work on memory effects in Bosonic channels [15. The contribution of VG to this work was supported by the European Community under contracts IST-SQUIBIT, IST-SQUBIT2, and RTN-Nanoscale Dynamics.
[1] S. L. Braunstein and A. K. Pati, Quantum Information Theory with Continuous Variables, (Kluwer, Dodrecht, 2001).

[2] C. H. Bennett and P. W. Shor, IEEE Trans. Inf. Theory 44, 2724 (1998); A. S. Holevo, arXiv:quant-ph/9809023

[3] V. Giovannetti, S. Guha, S. Lloyd, L. Maccone, J. H. Shapiro, and H. P. Yuen Phys. Rev. Lett. 92, 027902 (2004).

[4] C. H. Bennett, D. P. DiVincenzo, and J. A. Smolin, Phys. Rev. Lett. 78, 3217 (1997); C. King and M. B. Ruskai, IEEE Trans. Inf. Theory 47, 192 (2001).

[5] C. Macchiavello and G. M. Palma, Phys. Rev. A. 65, 050301(R) (2002); C. Macchiavello, G. M. Palma and S. Virmani, Phys. Rev. A. 69, 010303(R) (2004).

[6] G. Bowen and S. Mancini, Phys. Rev. A. 69, 012306 (2004); G. Bowen, I. Devetak and S. Mancini, arXiv:quant-ph/0312216

[7] J. Ball, A. Dragan and K. Banaszek, Phys. Rev. A 69, 042324 (2004); K. Banaszek, A. Dragan, W. Wasilewski, and C. Radzewicz, Phys. Rev. Lett. 92, 257901 (2004).

[8] C. M. Caves and P. D. Drummond, Rev. of Mod. Phys. 66, 481 (1994); H. P. Yuen and M. Ozawa, Phys. Rev. Lett. 70, 363 (1992).

[9] A. S. Holevo and R. F. Werner, Phys. Rev. A 63, 032312 (2001).
[10] V. Giovannetti, S. Guha, S. Lloyd, L. Maccone, J. H. Shapiro, B. J. Yen, and H. P. Yuen, in O. Hirota, ed. Quantum Information, Statistics, and Probability (Rinton Press, Princeton, 2004), pg. 90.

[11] D. F. Walls and G. J. Milburn, Quantum Optics (Springer-Verlag, Berlin, 1994).

[12] V. Giovannetti, S. Guha, S. Lloyd, L. Maccone, and J. H. Shapiro Phys. Rev. A 70, 032315 (2004); V. Giovannetti, S. Lloyd, L. Maccone, J. H. Shapiro, and B. J. Yen Phys. Rev. A 70 , 022328 (2004); V. Giovannetti and S. Lloyd, Phys. Rev. A 69, 062307 (2004).

[13] The right-hand side term of Eq. 23] is the asymptotic limit for $m \rightarrow \infty$ of the ratio between the maximum amount of classical information $C_{m}\left(\tilde{\mathcal{L}}^{\otimes m}, N\right)$ that can be reliably transmitted using $m$ copies of a channel whose map is $\tilde{\mathcal{L}}$. Clearly this cannot be smaller than the amount of classical information that one can transmit reliably by just using $\tilde{\mathcal{L}}$ once.

[14] A. S. Holevo, IEEE Trans. Inf. Theory 44, 269 (1998); B. Schumacher and M. D. Westmoreland, Phys. Rev. A 56, 131 (1997).

[15] N. J. Cerf, J. Clavareau, C. Macchiavello, and J. Roland, arXiv:quant-ph/0412089 